EPJ Web of Conferences 41, 05017 (2013)

DOI: $10.1051 /$ epjconf/20134105017

(C) Owned by the authors, published by EDP Sciences, 2013

\title{
Electronic Excited State and Vibrational Dynamics of Water Solution of Cytosine Observed by Time-resolved Transient Absorption Spectroscopy with Sub-10fs Deep Ultraviolet Laser Pules
}

\author{
Jun Miyazaki $^{1,2}$, Yuichiro Kida ${ }^{1,2}$, and Takayoshi Kobayashi ${ }^{1,2,3,4}$ \\ ${ }^{1}$ Advanced Ultrafast Laser Research Center, University of Electro-communications, 1-5-1 \\ Chofugaoka, Chofu, Tokyo 182-8585, Japan \\ ${ }^{2}$ JST, CREST, 5 Sanboncho, Chiyoda-ku, Tokyo 102-0075, Japan \\ ${ }^{3}$ Department of Electrophysics, National Chiao-Tung University, 1001 Ta Hsueh Rd., Hsinchu 300, \\ Taiwan \\ ${ }^{4}$ Institute of Laser Engineering, Osaka University, 2-6 Yamada-oka, Uuita, Osaka 565-0971, Japan
}

\begin{abstract}
Time-resolved transient absorption spectroscopy for water solution of cytosine with sub-10fs deep ultraviolet laser pulse is reported. Ultrafast electronic excited state dynamics and coherent molecular vibrational dynamics are simultaneously observed and their relaxation mechanisms are discussed.
\end{abstract}

\section{Introduction}

DNA and RNA exhibit ultrafast excited-state relaxation under deep ultraviolet (DUV) irradiation, which ensures that the molecules relax to the ground state through internal conversion before excited state reactions can occur. This scheme is considered to be crucial for photo-protection against mutagenic and carcinogenic effects in living systems. To understand excited state dynamics of biologically relevant molecules such as DNA bases (adenine, thymine, cytosine, and guanine) in the context of their photo stability, there have been many spectroscopic studies on such molecules by means of time-resolved absorption and fluorescence measurements. However, their excited state dynamics have not been fully understood yet due to the difficulty in generating ultrashort laser pulses in the DUV range.

In this study, sub 10-fs DUV pulse is successfully applied for the transient absorption spectroscopy of water solution of cytosine. DUV pulse with nearly Gaussian temporal and spectral profiles is generated by chirped pulse four-wave mixing technique without external compressor [1,2]. Transient absorption measurement using this ultrashort pulse allows us to simultaneously observe ultrafast electronic excited state dynamics and molecular vibrational dynamics.

\section{Experimental setup}

Sub-10 fs deep DUV pulse was used for pump-probe experiment. A part of the fundamental output from a Ti:Sapphire chirped pulse amplifier was focused on a $\mathrm{Kr}$-gas filled hollow fiber for spectral

This is an Open Access article distributed under the terms of the Creative Commons Attribution License 2.0, which permits unrestricted use, distribution, and reproduction in any medium, provided the original work is properly cited. 
broadening by the self-phase modulation. A near UV (NUV) pulse is also generated by frequency doubling of the fundamental pulse, which is negatively chirped by using a double-pass prism pair. The broadband near infrared (NIR) and NUV pulses were transmitted through another hollow fiber filled with Ar-gas, where a broadband DUV pulse was generated by the difference-frequency four wave mixing process. For transient absorption measurement and pulse characterization, the DUV pulse was spatially split into two at the edge of an aluminum mirror. One of the two beams was sent to an optical delay line composed of a stepping motor stage and used for probe pulse. The other beam was used for pump pulse. The pump and probe pulse were focus at the sample position by using a concave mirror. Time-resolved difference absorbance at 128 wavelengths in the spectral range from 4.22 to $4.86 \mathrm{eV}$ was measured simultaneously using a multichannel lock-in amplifier coupled to a polychrometer. The aqueous solution of cytosine was continuously circulated in a homemade flow cell by using a peristaltic pump to avoid photodegradation.

\section{Results and discussion}

(a)

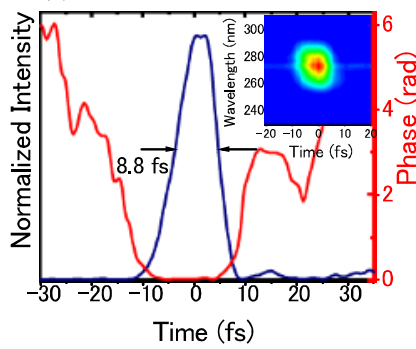

(b) $\triangle A>0$



(c)

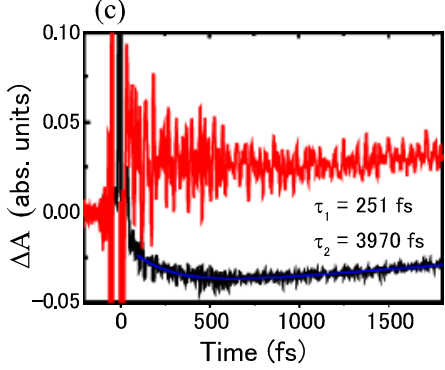

(d)

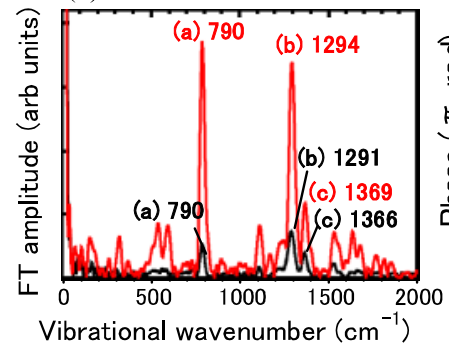

(e)

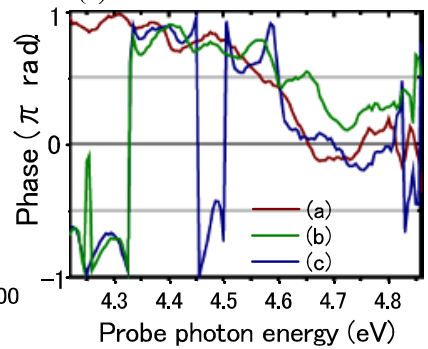

Fig. 1. (a) Retrieved intensity and phase of the DUV pulse. The pulse duration is $8.8 \mathrm{fs}$ in the full-width at half maximum. The inset shows the measured SD-FROG trace. (b) 2D difference transient absorption spectrum $\Delta A$ of aqueous solution of cytosine. $\Delta A$ is positive when the probe photon energy is below $\sim 4.45 \mathrm{eV}$, while that is negative above $\sim 4.45 \mathrm{eV}$. (c) Typical time traces of the difference absorbance in the low- (red line) and high-energy side (black line) in (b). $\Delta A$ is spectrally averaged between 4.30 and $4.36 \mathrm{eV}$ ( 4.65 and $4.75 \mathrm{eV}$ ) for the low- (high-)energy side. The blue line denotes two-exponential fitting curve for $\Delta A$ in the high-energy side with the time constants of 251 and $3970 \mathrm{fs}$. (d) Fourier amplitude spectra of the time trace in (c). (e) The phases of the observed molecular vibrations corresponding to the three modes in (d).

\subsection{The DUV laser pulse and stationary absorption}

The pulse duration of the DUV laser is measured using self-diffraction frequency-resolved optical gating (SD-FROG) technique. The commercial software, FROG 3.0 from Femtosoft Technologiesis, was employed for the analysis. Figure 1(a) shows the measured SD-FROG trace of DUV laser pulse and the retrieved temporal intensity. The retrieval error is 0.005 . The pulse duration is found to be 
$8.8 \mathrm{fs}$ in the full-width at half maximum. The inset shows the measured SD-FROG trace. The spectral peak of the DUV pulse is around $4.6 \mathrm{eV}$, which overlaps the absorption spectrum of cytosine at the edge.

\subsection{Dynamics of the electronic excited state}

Figure 1(b) shows the 2D difference absorption spectrum obtained by averaging two measurements. The experiments were performed at the intensities of the pump and probe pulses of 40 and $5 \mathrm{~nJ}$, respectively. $\Delta A$ is found to be positive when the probe photon energy is below $\sim 4.45 \mathrm{eV}$, while that is negative above $\sim 4.45 \mathrm{eV}$. Figure 1(c) show the typical time traces of $\Delta A$ in the high- and low-energy side, respectively. The intense oscillatory structures in the delay time between -100 fs and $100 \mathrm{fs}$ is attributed to electronic coherence induced by the two pulses. It is noteworthly that the observed signal is periodically modulated due to coherent molecular vibration. At the delay time longer than $100 \mathrm{fs}, \Delta A$ in the high-energy side decreases initially and then recovers slowly. The time trace of $\Delta A$ can be fitted by two exponentials with the time constants of $\sim 250$ fs and $\sim 4$ ps. Such multi-exponential decay dynamics has been observed in the previous transient absorption and fluorescence up conversion measurements $[3,4]$, and considered to be attributed to the excited state relaxation from $\left(\pi \pi^{*}\right)$ and $\left(n \pi^{*}\right)$ states, although vibrational population relaxation may also contribute. On the other hand, it is difficult to characterize the electronic excited state in the low-energy side because the signal is strongly modulated due to the molecular vibration even after $100 \mathrm{fs}$.

\subsection{Vibrational dynamics}

We calculated Fourier transform (FT) amplitude spectra of the time trace of $\triangle A$ to examine the origin of the measured molecular vibrational dynamic. Here, we analyzed $\Delta A$ after $100 \mathrm{fs}$ to exclude the initial contamination of the coherent artifact. Figure 1(d) shows FT amplitude of $\triangle A$ in the high- and low-energy side. Three intense vibrational peaks are observed, and peak positions in the high-energy side agree with that of the Raman frequency $(789,1289$, and 1364 $\mathrm{cm}^{-1}$ [5] , while that in high-energy side seems to be slightly blue-shifted. Figure 1(e) shows the phase of the observed molecular vibrations of three intense peaks as a function of probe photon energy. It appears that all three phases in the low-energy side are close to $\pi$. From this together with the sign of $\Delta A$ suggest that the signal in the low-energy side is mainly attributed to the excited state dynamics; the pump pulse excites cytosine to the electronic excited state, which is detected by the probe pulse predominantly as a transition to a higher excited state. The difference in the electronic configuration between the ground state and excited state may results in the difference in the measured vibrational frequencies.

\section{Conclusion}

Using sub-10 fs DUV pulses, the electronic excited state and vibrational dynamics of water solution of cytosine are simultaneously observed. The present study is expected to provide further understanding of relevant biological molecules such as DAN and gives some insight on the ultrafast photochemical dynamics induced by DUV irradiation.

1. Y. Kida and T. Kobayashi, J. Opt. Soc. Am. B. 28, 139 (2011)

2. T. Kobayashi and Y. Kida, Phys. Chem. Chem. Phys.14, 6193 (2012)

3. A. Sharonov, T. Gustavsson, V. Carré, E. Renault, and D. Markovitsi, Chem. Phys. Lett. 380, $173(2003)$

4. P. M. Hare, C. E. Crespo-Hernández, and B. Kohler, Proc. Natl. Acad. Sci. 104, 435 (2007)

5. B. E. Billinghurst, S. A. Oladepo and G. R. Loppnow, J. Phys. Chem. B 113, 7392 (2009) 